\title{
AN ELEMENTARY PROOF OF A RESULT ON $\Lambda(p)$ SETS
}

\author{
KATHRYN E. HARE
}

(Communicated by William J. Davis)

\begin{abstract}
We give an elementary proof of the fact that a subset of the dual of a compact abelian group which is a $\Lambda(p)$ set for some $1 \leq p<2$ is a $\Lambda(p+\varepsilon)$ set for some $\varepsilon>0$, and extend this result to $\Lambda(p)$ sets for $0<p<1$.
\end{abstract}

Let $G$ be a compact abelian group with normalized Haar measure $m$, and let $\hat{G}$ be its discrete dual group. As in [6] we say that $E \subset \hat{G}$ is a $\Lambda(p)$ set for some $p>0$ provided there exists $0<q<p$ and a constant $c=c(p, q, E)$ such that

$$
\|f\|_{p} \equiv\left(\int|f|^{p} d m\right)^{1 / p} \leq c\|f\|_{q}
$$

whenever $f \in \operatorname{Trig}_{E} G \equiv\{f: G \rightarrow \mathbf{C}: \operatorname{supp} \hat{f}$ is a finite subset of $E\}$. The least such constant $c$ is called the $\Lambda(p, q)$ constant of $E$ and is denoted by $\Lambda(p, q, E)$. An application of Hölder's inequality shows that if $(*)$ holds for some $q<p$ then it holds for all such $q$, with different values for $c$. Moreover, if $E$ is a $\Lambda(p)$ set and $r<p$, then $E$ is a $\Lambda(r)$ set. For standard results on $\Lambda(p)$ sets we refer the reader to $[6$ or 3$]$.

In [1] Bachelis and Ebenstein showed that if $E$ is a $\Lambda(p)$ set for some $1 \leq p<2$, then $E$ is a $\Lambda(p+\varepsilon)$ set for some $\varepsilon>0$. Their proof was based on a deep result by Rosenthal [5]. In this note we give an elementary, self-contained proof of this fact about $\Lambda(p)$ sets and extend it to $\Lambda(p)$ sets with $0<p<1$.

THEOREM. Let $0<p<2$ and suppose $E \subset \hat{G}$ is a $\Lambda(p)$ set. Then $E$ is a $\Lambda(q)$ set for some $q>p$.

For the proof we need the following three lemmas.

LEMMA 1. Suppose $E$ is a $\Lambda(p)$ set, $p>0$, with $\Lambda(p, p / 2, E) \leq c$. Let $n \geq 4 c^{p}$. Then for every nonzero $f \in \operatorname{Trig}_{E} G$ and for every $A \subset G$ with $m(A) \leq 1 / n$,

$$
\int_{A}|f|^{p} d m<\left(1-\frac{1}{n}\right)\|f\|_{p}^{p}
$$

Proof. Suppose there is some nonzero $f \in \operatorname{Trig}_{E} G$ and $A \subset G$ with $m(A) \leq$ $1 / n$ such that

$$
\int_{A}|f|^{p} \geq\left(1-\frac{1}{n}\right)\|f\|_{p}^{p}
$$

Received by the editors September 3, 1987 and, in revised form, January 18, 1988.

1980 Mathematics Subject Classification (1985 Revision). Primary 43A46.

Research partially supported by NSERC Grant A9333. 
Without loss of generality we may assume $\|f\|_{p}=1$. By Hölder's inequality

$$
\int_{A}|f|^{p / 2} \leq m(A)^{1 / 2}\|f\|_{p}^{p / 2} \leq \frac{1}{\sqrt{n}}
$$

Also

Hence

$$
\int_{A^{c}}|f|^{p / 2} \leq\left(\int_{A^{c}}|f|^{p}\right)^{1 / 2}<\frac{1}{\sqrt{n}}
$$

$$
\|f\|_{p / 2}<(2 / \sqrt{n})^{2 / p}\|f\|_{p} \leq(1 / c)\|f\|_{p}
$$

which contradicts the assumption that $\Lambda(p, p / 2, E) \leq c$.

Let $p, q, \lambda>0$ and $n \in \mathbf{Z}$. We will say that $E \subset \hat{G}$ has property $P(p, q, \lambda, n)$ if there are $f_{1}, \ldots, f_{n} \in \operatorname{Trig}_{E} G$ such that

(1) $\left\|f_{i}\right\|_{p}=1$ for $i=1, \ldots, n$, and

(2) $\left\|\sum_{i=1}^{n} \alpha_{i} f_{i}\right\|_{p} \geq \lambda\left(\sum_{i=1}^{n}\left|\alpha_{i}\right|^{q}\right)^{1 / q}$ for all scalars $\alpha_{1}, \ldots, \alpha_{n}$.

LEMMA 2. Suppose $\lambda, \delta>0,0<p<2$ and $n \in \mathrm{Z}$. There is an $N=$ $N(\lambda, \delta, p, n)$ so that if $E$ has property $P(p, p, \lambda, N)$, then there are disjoint measurable sets $A_{1}, \ldots, A_{n} \subset G$ and nonzero $f_{1}, \ldots, f_{n} \in \operatorname{Trig}_{E} G$ such that

$$
\int_{A_{i}}\left|f_{i}\right|^{p} \geq \frac{\lambda^{2 p /(2-p)}}{1+\delta}\left\|f_{i}\right\|_{p}^{p}
$$

for all $i=1, \ldots, n$.

REMARKS. 1. It is proven in [2] and [4] that if $E$ has property $P(p, p, \lambda, N)$ then there are disjoint measurable sets $A_{1}, \ldots, A_{N} \subset G$ and nonzero $f_{1}, \ldots, f_{N} \in$ $\operatorname{Trig}_{E} G$ such that $\int_{A_{i}}\left|f_{i}\right|^{p} \geq \lambda^{2 p /(2-p)}\left\|f_{i}\right\|_{p}^{p}$ for $i=1, \ldots, N$. The proof of this stronger result is not as elementary as the one we give for Lemma 2.

2. The first part of the proof we give below is from [2]. We present it here for completeness. The second part is a modification of a known argument (cf. [7, VII]).

Proof. Suppose $f_{1}, \ldots, f_{N} \in \operatorname{Trig}_{E} G$ and satisfy (1) and (2). Let $r_{i}(t)$ be the Rademacher functions. By (2), averaging over $t$ using Fubini's theorem, and applying Hölder's inequality, we obtain

$$
\begin{aligned}
\lambda^{p} N & \leq \int_{0}^{1}\left\|\sum_{i=1}^{N} r_{i}(t) f_{i}(x)\right\|_{p}^{p} d t=\int_{0}^{1} \int_{0}^{1}\left|\sum_{i=1}^{N} r_{i}(t) f_{i}(x)\right|^{p} d t d x \\
& \leq \int_{0}^{1}\left(\int_{0}^{1}\left|\sum_{i=1}^{N} r_{i}(t) f_{i}(x)\right|^{2} d t\right)^{p / 2} d x=\int_{0}^{1}\left(\sum_{i=1}^{N}\left|f_{i}(x)\right|^{2}\right)^{p / 2} d x \\
& \leq \int_{0}^{1}\left(\max _{1 \leq i \leq N}\left|f_{i}(x)\right|^{2-p}\right)^{p / 2}\left(\sum_{i=1}^{N}\left|f_{i}(x)\right|^{p}\right)^{p / 2} d x \\
& \leq\left(\int_{0}^{1}\left(\max _{1 \leq i \leq N}\left|f_{i}(x)\right|^{p} d x\right)\right)^{(2-p) / 2}\left(\int_{0}^{1}\left(\sum_{i=1}^{N}\left|f_{i}(x)\right|^{p}\right) d x\right)^{p / 2} \\
& =N^{p / 2}\left(\int_{0}^{1} \max _{i}\left|f_{i}(x)\right|^{p} d x\right)^{(2-p) / 2} .
\end{aligned}
$$


Thus

$$
\int_{0}^{1} \max _{1 \leq i \leq N}\left|f_{i}(x)\right|^{p} d x \geq \lambda^{2 p /(2-p)} N
$$

For $i=1, \ldots, N$ let

$$
A_{i}=\left\{x: i \text { is the first index } j \text { such that }\left|f_{j}(x)\right|=\max _{1 \leq k \leq N}\left|f_{k}(x)\right|\right\} .
$$

Obviously the $A_{i}$ 's are disjoint. Moreover,

$$
\lambda^{2 p /(2-p)} N \leq \sum_{i=1}^{N} \int_{A_{i}} \max _{1 \leq j \leq N}\left|f_{j}(x)\right|^{p} d x=\sum_{i=1}^{N} \int_{A_{i}}\left|f_{i}\right|^{p} d x .
$$

Set

$$
J=\left\{i: \int_{A_{i}}\left|f_{i}\right|^{p} d x \geq \frac{\lambda^{2 p /(2-p)}}{1+\delta}\right\}
$$

Then

$$
\lambda^{2 p /(2-p)} N \leq|J|+\frac{\lambda^{2 p /(2-p)} N}{1+\delta} .
$$

So $|J| \geq N \lambda^{2 p /(2-p)} \delta /(1+\delta) \geq n$ if $N$ is sufficiently large.

For part of the proof of Lemma 3 we use arguments similar to some found in [5, Lemma 6].

LEMMA 3. Let $0<p<q$. If $E$ is not a $\Lambda(q)$ set then $E$ has property $P(p, q, \lambda, n)$ for every $0<\lambda<1$ and integer $n$.

ProOF. Fix $0<\lambda<1$ and $n \in \mathbf{Z}$. If $q>1$ let $q^{\prime}=q /(q-1)$ and choose $0<\delta<1$ so that $\delta-n^{1 / q^{\prime}}\left(1-\delta^{q}\right)^{1 / q} \geq \lambda$. For $q \leq 1$ choose $0<\delta<1$ satisfying $2 \delta^{q}-1 \geq \lambda^{q}$.

Since $E$ is not a $\Lambda(q)$ set we may choose a finite subset $F$ of $E$ with $\Lambda(q, p, F) \equiv$ $M>\left((n+1) /\left(1-\delta^{q}\right)\right)^{1 / p}$. Select $f_{1} \in \operatorname{Trig}_{F} G$ so that $\left\|f_{1}\right\|_{p}=1$ and $\left\|f_{1}\right\|_{q}=M$. Let $A_{1}=\left\{x:\left|f_{1}(x)\right|>M\right\}$. Then $m\left(A_{1}\right) \leq M^{-p}$,

$$
\int_{A_{1}^{c}}\left|f_{1}\right|^{q} \leq M^{q-p} \int_{A_{1}^{c}}\left|f_{1}\right|^{p} \leq M^{q-p}
$$

and

$$
\int_{A_{1}}\left|f_{1}\right|^{q} \geq M^{q}-M^{q-p}>\delta^{q} M^{q} .
$$

(The final inequality is true because of the choice of $M$.)

Now assume inductively that for some $1 \leq k \leq n$ there exist $f_{1}, \ldots, f_{k} \in \operatorname{Trig}_{F} G$ and disjoint measurable sets $A_{1}, \ldots, A_{k} \subset G$, with each $f_{i}$ a translate of $f_{1}$, $m\left(A_{i}\right) \leq M^{-p}$, and $\left\|f_{i} 1_{A_{i}}\right\|_{q}>\delta M$.

Let $B=\bigcup_{1}^{k} A_{i}$. Then $m\left(B^{c}\right) \geq 1-k M^{-p} \geq 1-n M^{-p}$. Also, if we let $f_{g}(x)=f_{1}(x-g)$, then Fubini's theorem shows that

$$
\int_{G} \int_{B^{c}}\left|f_{g}(x)\right|^{q}=\int_{B^{c}}\left\|f_{1}\right\|_{q}^{q} \geq M^{q}\left(1-n M^{-p}\right) .
$$


Hence there exists some $g \in G$ such that for $f_{k+1} \equiv f_{g}, \int_{B^{c}}\left|f_{k+1}\right|^{q} \geq$ $M^{q}\left(1-n M^{-p}\right)$. Set $A_{k+1}=\left\{x:\left|f_{k+1}(x)\right|>M\right\} \cap B^{c}$. Then $m\left(A_{k+1}\right) \leq M^{-p}$, $A_{k+1} \cap A_{i}=\varnothing$ for $i \leq k$ and

$$
\int_{B^{c} \backslash A_{k+1}}\left|f_{k+1}\right|^{q} \leq M^{q-p} \int_{B^{c} \backslash A_{k+1}}\left|f_{k+1}\right|^{p} \leq M^{q-p} .
$$

Therefore

$$
\int_{A_{k+1}}\left|f_{k+1}\right|^{q} \geq M^{q}\left(1-n M^{-p}\right)-M^{q-p}>\delta^{q} M^{q}
$$

which completes the induction step.

Now let $\varphi_{i}=f_{i} 1_{A_{i}}$ for $i=1, \ldots, n$. Then

$$
\left\|\varphi_{i}-f_{i}\right\|_{q}^{q}=\int_{A_{i}^{q}}\left|f_{i}\right|^{q} \leq M^{q}\left(1-\delta^{q}\right) .
$$

If $q \leq 1,(* *)$, together with the triangle inequality, shows that for any choice of scalars $\alpha_{1}, \ldots, \alpha_{n}$,

$$
\left\|\sum_{i=1}^{n} \alpha_{i}\left(\varphi_{i}-f_{i}\right)\right\|_{q}^{q} \leq \sum_{i=1}^{n}\left|\alpha_{i}\right|^{q} M^{q}\left(1-\delta^{q}\right)
$$

while if $q>1,(* *)$, the triangle inequality and Hölder's inequality combine to give

$$
\left\|\sum \alpha_{i}\left(\varphi_{i}-f_{i}\right)\right\|_{q} \leq\left(\sum_{i=1}^{n}\left|\alpha_{i}\right|^{q}\right)^{1 / q} n^{1 / q^{\prime}} M\left(1-\delta^{q}\right)^{1 / q} .
$$

Since $A_{1}, \ldots, A_{n}$ are disjoint it follows that if $q \leq 1$,

$$
\begin{aligned}
\left\|\sum_{i=1}^{n} \alpha_{i} f_{i}\right\|_{q}^{q} & \geq\left\|\sum \alpha_{i} \varphi_{i}\right\|_{q}^{q}-\left\|\sum \alpha_{i}\left(\varphi_{i}-f_{i}\right)\right\|_{q}^{q} \\
& \geq \sum_{i=1}^{n} \int_{A_{i}}\left|\alpha_{i} f_{i}\right|^{q}-\sum_{i=1}^{n}\left|\alpha_{i}\right|^{q} M^{q}\left(1-\delta^{q}\right) \\
& \geq \sum_{i=1}^{n}\left|\alpha_{i}\right|^{q} M^{q}\left(\delta^{q}-\left(1-\delta^{q}\right)\right) \\
& =\sum_{i=1}^{n}\left|\alpha_{i}\right|^{q} M^{q} \lambda^{q}
\end{aligned}
$$


while if $q>1$,

$$
\begin{aligned}
\left\|\sum_{i=1}^{n} \alpha_{i} f_{i}\right\|_{q} & \geq\left\|\sum \alpha_{i} \varphi_{i}\right\|_{q}-\left\|\sum \alpha_{i}\left(\varphi_{i}-f_{i}\right)\right\|_{q} \\
& \geq\left(\sum_{i} \int_{A_{i}}\left|\alpha_{i} f_{i}\right|^{q}\right)^{1 / q}-\left(\sum\left|\alpha_{i}\right|^{q}\right)^{1 / q} n^{1 / q^{\prime}} M\left(1-\delta^{q}\right)^{1 / q} \\
& \geq\left(\sum_{i}\left|\alpha_{i}\right|^{q}\right)^{1 / q} M\left(\delta-n^{1 / q^{\prime}}\left(1-\delta^{q}\right)^{1 / q}\right) \\
& \geq\left(\sum_{i}\left|\alpha_{i}\right|^{q}\right)^{1 / q} M \lambda .
\end{aligned}
$$

But the choice of $F$ ensures that

$$
\left\|\sum \alpha_{i} f_{i}\right\|_{p} \geq \frac{1}{M}\left\|\sum \alpha_{i} f_{i}\right\|_{q}
$$

hence in either case

$$
\left\|\sum_{i=1}^{n} \alpha_{i} f_{i}\right\|_{p} \geq \lambda\left(\sum_{i=1}^{n}\left|\alpha_{i}\right|^{q}\right)^{1 / q}
$$

for all scalars $\alpha_{1}, \ldots, \alpha_{n}$ and so $E$ has property $P(p, q, \lambda, n)$.

PROOF OF THE THEOREM. Suppose $E$ is a $\Lambda(p)$ set with $c=\Lambda(p, p / 2, E)$. Let $n$ be an integer with $n \geq 4 c^{p}$, let $\lambda=\left(1-1 / n^{2}\right)^{(2-p) / 2 p}$ and let $N=N(\lambda, 1 / n, p, n)$ as in Lemma 2.

If $E$ has property $P(p, p, \lambda, N)$ then by Lemma 2 there are disjoint measurable sets $A_{1}, \ldots, A_{n} \subset G$ and nonzero $f_{1}, \ldots, f_{n} \in \operatorname{Trig}_{E} G$ such that

$$
\int_{A_{i}}\left|f_{i}\right|^{p} \geq\left(\frac{1-1 / n^{2}}{1+1 / n}\right)\left\|f_{i}\right\|_{p}^{p}=(1-1 / n)\left\|f_{i}\right\|_{p}^{p}
$$

for all $i=1, \ldots, n$. For some $i, m\left(A_{i}\right) \leq 1 / n$ and this contradicts Lemma 1 . Thus $E$ cannot have property $P(p, p, \lambda, N)$.

Let $q>p$ satisfy $N^{1 / q-1 / p} \geq \sqrt{\lambda}$ and suppose $E$ has property $P(p, q, \sqrt{\lambda}, N)$. Assume $f_{1}, \ldots, f_{N} \in$ Trig $_{E} G$ satisfy

(1) $\left\|f_{i}\right\|_{p}=1$ for $i=1, \ldots, N$, and

(2) $\left\|\sum_{i=1}^{N} \alpha_{i} f_{i}\right\|_{p} \geq \sqrt{\lambda}\left(\sum_{i=1}^{N}\left|\alpha_{i}\right|^{q}\right)^{1 / q}$ for all scalars $\alpha_{1}, \ldots, \alpha_{N}$.

By Hölder's inequality

$$
\left(\sum_{i=1}^{N}\left|\alpha_{i}\right|^{p}\right)^{1 / p} \leq\left(\sum_{i=1}^{N}\left|\alpha_{i}\right|^{q}\right)^{1 / q} N^{1 / p-1 / q}
$$

thus

$$
\begin{aligned}
\left\|\sum_{i=1}^{N} \alpha_{i} f_{i}\right\|_{p} & \geq N^{1 / q-1 / p} \sqrt{\lambda}\left(\sum_{i=1}^{N}\left|\alpha_{i}\right|^{p}\right)^{1 / p} \\
& \geq \lambda\left(\sum_{i=1}^{N}\left|\alpha_{i}\right|^{p}\right)^{1 / p}
\end{aligned}
$$


This means that $E$ has property $P(p, p, \lambda, N)$ which contradicts our work above. Hence $E$ fails to have property $P(p, q, \sqrt{\lambda}, N)$, and so by Lemma 3 is a $\Lambda(q)$ set.

REMARK. Our method of proof can be used to show that if $E$ is a $\Lambda(p)$ set for some $0<p<2$, with $\Lambda(p, p / 2, E) \leq c$, then $E$ is a $\Lambda(q)$ set with

$$
\Lambda(q, p, E) \leq \begin{cases}\left(\frac{32 p n^{4}}{2-p}\right)^{q / p} & \text { if } p \geq 1 \\ \left(\frac{32 n^{4}}{2-p}\right)^{1 / p} & \text { if } p<1\end{cases}
$$

where $n \in \mathbf{Z}, n \geq 4 c^{p}$ and

$$
\frac{1}{p}>\frac{1}{q} \geq \frac{1}{p}+\frac{2-p}{4 p} \frac{\log \left(1-1 / n^{2}\right)}{\log 2 n^{2}} .
$$

COROLlaRY. $L_{E}^{1}$ is reflexive if and only if $E$ is a $\Lambda(1)$ set.

ProOF. If $E$ is a $\Lambda(1)$ set then $E$ is a $\Lambda(p)$ set for some $p>1$. Hence $L_{E}^{1}=L_{E}^{p}$ and thus is reflexive.

If $L_{E}^{1}$ is reflexive then the unit ball of $L_{E}^{1}$ is uniformly integrable. Hence there exists $\delta>0$ so that whenever $m(A)<\delta$ and $f \in L_{E}^{1}$ then $\int_{A}|f| \leq \frac{1}{2}\|f\|_{1}$.

Let $f \in \operatorname{Trig}_{E} G$ with $\|f\|_{1}=1$. Let $A=\{x:|f(x)|>1 / \delta\}$. Then $m(A)<\delta$ so $\int_{A}|f| \leq \frac{1}{2}$ and hence $\int_{A^{c}}|f| \geq \frac{1}{2}$. Thus

$$
\|f\|_{1 / 2} \geq\left(\int_{A^{c}}|f|^{1 / 2}\right)^{2} \geq\left(\sqrt{\delta} \int_{A^{c}}|f|\right)^{2} \geq \frac{\delta}{4}\|f\|_{1}
$$

and since $f$ was arbitrary $E$ is a $\Lambda(1)$ set.

The author would like to thank Dr. Tomczak-Jaegermann for a number of helpful discussions.

\section{REFERENCES}

1. G. Bachelis and S. Ebenstein, On $\Lambda(p)$ sets, Pacific J. Math. 54 (1974), 35-38.

2. L. Dor, On projections in $L_{1}$, Ann. of Math. (2) 102 (1975), 463-474.

3. J. Lopez and K. Ross, Sidon sets, Lecture Notes in Pure Appl. and Math., vol. 13, Marcel Dekker, New York, 1975.

4. B. Maurey, Projections dans $L^{1}$, 'd'apres L. Dor', is part of the title. Sem. Maurey-Schwartz 1974/75, \#21.

5. H. P. Rosenthal, On subspaces of $L^{p}$, Ann. of Math. (2) 97 (1973), 344-373.

6. W. Rudin, Trigonometric series with gaps, J. Math. Mech. 9 (1960), 203-227.

7. N. Tomczak-Jaegermann, Finite-dimensional operator ideals and Banach-Mazur distances, Pitman, Boston, Mass. (to appear).

Department of Mathematics, University of Alberta, Edmonton, Alberta, CANADA V6G 2G1

Current address: Department of Pure Mathematics, University of Waterloo, Waterloo, Ontario, Canada, N2L3G1 